\title{
Field Theory in Practice
}

\author{
José Méndez Omaña ${ }^{1}$, \\ ${ }^{1}$ Beuth-Hochschule für Technik Berlin \\ Berlin, Germany \\ mendez@beuth-hochschule.de
}

\begin{abstract}
The aim of this paper is to present K. Lewin's Field Theory in practice, applying it to evacuation simulations. The construction of the field is discussed from the scratch in order to define the psychological space of a social event. Then, we illustrate the interplay of the physical and the psychological aspects of a mathematical model of human behavior presenting and discussing evacuation simulations of one event. At first we simulate the physical situation and then we calculate an evacuation time applying an elementary microscopic model. An empirical plausibility control is presented. When we apply mathematical modeling or cybernetic methods to social sciences we are said to be careful. Nevertheless, a social event might be considered, on a natural way, as a set of time series, from the point of view of statistics. In this paper we restrict ourselves to introduce probabilities of random variables associated to the physical and the psychological space and define a measure for the evacuation simulation: The entropy of the event, merging the physical and the psychological spaces.
\end{abstract}

Keywords: Field Theory, Mathematical Models of Human Behaviour, Social Event Entropy

\section{Introduction}

Any mathematical model applied in evacuation simulations includes, or should do, implicitly or explicitly, psychological aspects due to its purpose: modelling human behaviour. Data and mathematical concepts are required, but we are told: "For a good statistic of society, we need long runs under essentially constant conditions" [12]. This statement of N. Wiener, inventor of cybernetics, continues: "Thus the human sciences are very poor testing-grounds for a new mathematical technique". We are in the need of interdisciplinary expertise. This is the reason why, Lewin's field theory is interesting. K. Lewin was a psychologist who used mathematical theory, set-theoretic topology and vectors, to express human behaviour [4] and, as we reportedly know, participated in meetings organised by Wieners work group [12]. Concerning pedestrian dynamics the link to psychology exhibits a diversity of forms. For instance: Helbing's Social Force Model [2] includes explicit reference to the work of Lewin and the hydraulic model of Predtetschenski and Milinski [8] presents an implicit solution based on empirics. Let us argue briefly about this assertion: When we work out a task applying this method, the working parameters may suggest psychological aspects play no role. Nevertheless, when the authors introduce the velocity as a function of density and sort of way, they assert that for the same density (on the same track) the values of the velocity may differ strongly and this because of the individual characteristics of each person. The authors introduce explicitly an extended definition of the velocity as a function of three variables: density, sort of way and psychological factors. Nevertheless, for the calculations, we find the velocity as univariate function of the density. Almost 3600 empirical measurements and a statistical data treatment justify the simplification [8]. The question about a good statistic of society rises from at least two points of view and concerns not only this model: Do we have long runs under essentially equal conditions? If we have a good statistic of one society, is it a good statistic of another society?

An important discussion of some psychological aspects of evacuation simulation can be found in [10]. 
The paper consists of two sections: Field Theory and Introducing Entropy. In the first section we present some basics of Lewin's Field Theory aiming immediate application. In order to accomplish this object and according to the material bounds of this paper one simple case study is presented. The aim of the second section is the introduction of a kind of measurement of a social event: the concept of social event entropy. This happens following strictly Shannon's idea: to define a measurement for uncertainty [11].

\section{Field Theory}

Field theory is a method of analysing causal relations and of building scientific constructs (systems of a priori concepts) and can be expressed in the form of certain general statements about the nature of the conditions of change of the life or psychological space of a person [6]. Behaviour is any change in the psychological space. A basic construct states that the behaviour $b$ at the time $t$ is a function of the situation $S$ at the time $t$ and only and this time. This can be written as follows: $\mathrm{b}_{\mathrm{t}}=\mathrm{F}\left(\mathrm{S}_{\mathrm{t}}\right)$. Thus, modelling the behaviour of a person requires defining her/his life space at a given time. The life space of a person is the totality of possible psychological events. Another expression for behaviour in Lewin's papers is $B=f(P E)$, behaviour as a function of the state of the person an of the environment [4].

In order to explain the constructive procedure of representing the life space of a person and its environment we start excerpting from [4] and later present a rather schematic procedure:

1. "The fundamental constructs which we use in representing the situation must consist of concepts from which one can derive, unambiguously, certain events as "possible", others as "not possible". Instead of classificatory concepts one has to use constructive ones which have a direct relationship to laws."

2. "It should be possible to derive from such a representation all forms of anamneses which actually occur. This stringency of the derivation of the totality of possible cases is valid not only for the anamneses of the person within the situation but also for the possible changes of the person or the situation itself."

3. "Such a derivation of the totality of possibilities can only be accomplished if one proceeds from the life space as a whole."

4. "The center of interest shifts from objects to processes, from states to changes of state. If the life space is a totality of possible events, then "things" that enter the situation, especially the person himself and psychological "objects", have to be characterized by their relationship to possible events."

An important remark has to be made: if we read possible case, then we eventually associate a probability to this case. In this context we have to be aware of the theory and consider that only the subjective probability should be taken into account for predicting behaviour [6]. This means, only what a person knows, plays a role.

In the following we shall speak only about psychological space including the life space concept. As far as we know, the distinction is of historical nature. In the early papers Lewin speaks about life space and psychological space, later about psychological and hodological space.

The rather formal aspects of field theory as the comparison of the mathematical concept of topological space with the hodological space concept might be found in [4] or [7]. Here we merely mention that the Euclidian space concept, say the everyday space with the usual distance, is not suited to describe psychological events. The hodological space is a concept representing an empirical (psychological) space. This is a diversity of facts showing specific forms of interaction at some time [5]. Etymologically explained, hodological space means something like the space where you find the paths (Greek: ódós, path, way). The hodological space is set consisting of regions also called cells. The regions may be adjacent or not and they may contain subregions. The regions represent domains of the psychological world of a person or a group of persons. Two regions are said to be independent if a change in one of them does not induce a change in the other one. A point is not a region. Two points may be connected by a path and direction and distance are defined be specified paths. The distance between two regions $a$ and $b$ is defined as the minimal number of regions crossed by a path from $a$ to $b$. There is also a concept of connectivity as in topology. A change of position in the hodological space is called locomotion and corresponds to the 
behaviour. It should be considered at a given time. At this point we have to say, that in the field theory a given time $t$ means often an interval of time and not a point on the time scale. The reason why: we need to know the context of the psychological event and this is practically impossible if we consider only an instant. In practice we could also speak about frames but we are not going to do it.

Before we present a schematic procedure for the psychological space set up, we would like to say that, from the point of view of psychology, there are two approaches for this task: the one is of historical nature, anamneses, and the other diagnostic tests of the present. The first one can't be applied in the standard event management and moreover, following Lewin, won't produce the desired results. Hence, we propose a diagnostic test procedure:

Step 1 Enter the time interval $[0, T]$, the total event time duration. Define the length of the time subintervals for diagnostic test and determine, if needed, a frequency.

Step 2 Define the relevant variables.

Step 3 For the first subinterval: attempt to find an answer to the question: What is possible? (Based on the actual situation)

Step 4 For the first subinterval: proof the values of the relevant variables

Step 5 For the first subinterval: sketch the hodological space

Step 6 Return to Step 3 for the second subinterval and so on until required.

We finish this part of this section with two remarks: the proof of the relevant variables may have consequences in the physical world and vice versa. The second remark is, may be, much more than a remark: The diagnostic tests of the present includes elements of the past as the 'habits' of the visitors. This is well known to all event managers.

\subsection{Field theory in practice: Example}

We apply a quite simple microscopic model developed on our own. For a solution to this kind of problems based on a force field model see [1]. The estimated path of each person or group of persons is given by a curve. Beziér-Curves or splines are in some cases useful. The direction field of a linear, first order ODE with constant coefficients is, in other cases, good suited, because it is easy to obtain solutions for given boundary values. Some of the solutions are used as boundary paths of the pedestrian flow and some other represent special paths. The adequate kind of mathematical objects depends on the geometry and specific characteristics of the venue and event. The area bounded by the boundary paths is calculated and, depending on the flow, we estimate the density at a given time. The quotient of the arc length of a curve or a differential equation solution and a locally suited velocity, according to the density, provides a time estimate.

We illustrate the method by a very simple example: a group of 56 persons, 55 students, one of them using a wheelchair and requiring personal assistance, evacuates a hall. We focus only on the last part of the evacuation namely the specific flow through the door and the flow on the area in front of the door. The width of the door amounts $1.09 \mathrm{~m}$ and we consider $2 \mathrm{~m}$ in front of. Because of the extension limit of this paper we omit a sketch but the Fig.1 may fill the gap: Imagine the door between the upper right corner, this is the point with the coordinates $(2,0)$, and the point $(2,-1.09)$. From this point downwards to $(2,-3)$ we have a wall. The upper horizontal axis should be seen as a wall. The remaining lines, from $(0,0)$ to $(0,-3)$ and from this point to $(2,-3)$ are open, inside of the hall. We choose the direction field of the differential equation $y^{\prime}=0.3 x-y$ on the closed interval $[0,2]$ for the independent variable $\mathrm{x}$ and on $[-3$, $0.04]$ for $y$. The reason why is the geometry of this special part of the track: a relatively short non-linear path showing eventually high density. The exact solution of this equation is $\mathrm{y}=\mathrm{C} e^{-x}+0.3 x-0.3$, with $\mathrm{C}=-2.22$ for the boundary condition $\mathrm{y}(2)=0, \mathrm{C}=-10.12$ for $\mathrm{y}(2)=-1.07$. This are the solutions used as bounds for the pedestrian flow. The area enclosed by the graphs of this functions, the bottom horizontal line passing through $(0,-3)$ and the perpendicular to this line passing through $(2,0)$ amounts $3.22 \mathrm{~m}^{2}$. A third solution $\mathrm{C}=-6.65$ for $\mathrm{y}(2)=-0.6$ is given, in order to simulate a frequently used path towards and through a point slightly to the right of the midpoint of the threshold of the door. The arc lengths amount $3.28 \mathrm{~m}$ and $2.08 \mathrm{~m}$ for the upper and for the lower bound respectively. The third arc length amounts 2.71 
$\mathrm{m}$. The shape of the pedestrian flow shall be "narrow, wide, narrow", expressed on a qualitative way, based on empirical knowledge. Quantitatively we assume the pattern $1+1+1+2+2+3$ persons, then 9 times 4 and lastly $3+3+2+1+1$. For the first three we set $v=1.34+0.26=1.6 \mathrm{~m} / \mathrm{s}$ obtaining $\mathrm{t}=1.69 \mathrm{~s}$ following the path in the middle. For the next four persons we set $\mathrm{v}=1.34 \mathrm{~m} / \mathrm{s}$, also on the path in the middle, and this yields to $t=2.02 \mathrm{~s}$. We are aware of the pattern, this means: after $3.71+\mathrm{e}=1.69+2.02+\mathrm{e}$ seconds, where e is an error, 7 persons went along the path in the middle, due to the low density. We recall: the door width amounts $1.09 \mathrm{~m}$. If we assume that, in average, less than two persons but more than one may cross the threshold of the door, then, from now on, the flow sticks in traffic. Moreover, we dare to make a first prediction: If 1.5 persons per second would cross the threshold of the door then we estimate $t_{1}=56 / 1.5=37.3 \mathrm{~s}$ for the whole group. After this remarks we continue the calculation: For the coming three persons we set $\mathrm{v}=1.23 \mathrm{~m} / \mathrm{s}$ and we have $\mathrm{t}=2.51 \mathrm{~s}$. For the four persons groups we set $\mathrm{v}=1.11 \mathrm{~m} / \mathrm{s}$ and calculate two rather on the upper bound $t_{1}=3.28 / 1.11=2.95 \mathrm{~s}$ and two rather on the lower bound obtaining $\mathrm{t}_{2}=2.08 / 1.11=1.87 \mathrm{~s}$. The mean of this values is $\mathrm{t}=2.41 \mathrm{~s}$.

We add up and obtain $3.71+2.51+9 \cdot 2.41=27.9 \mathrm{~s}$ and 46 persons. For the last groups consisting of three persons we set $\mathrm{t}=2 \cdot 2.51=5.02 \mathrm{~s}$, hence we have until now $\mathrm{t}=32.92 \mathrm{~s}$ and 52 persons. For the group consisting of two persons, one of them on the wheelchair we set $v=0.8 \mathrm{~m} / \mathrm{s}$ on the path in the middle and obtain $t=3.39 \mathrm{~s}$. The last two persons have to adapt their individual velocities to the one of the persons there are behind of and define in fact a two persons group. The result is $t=39.7+e$ s.

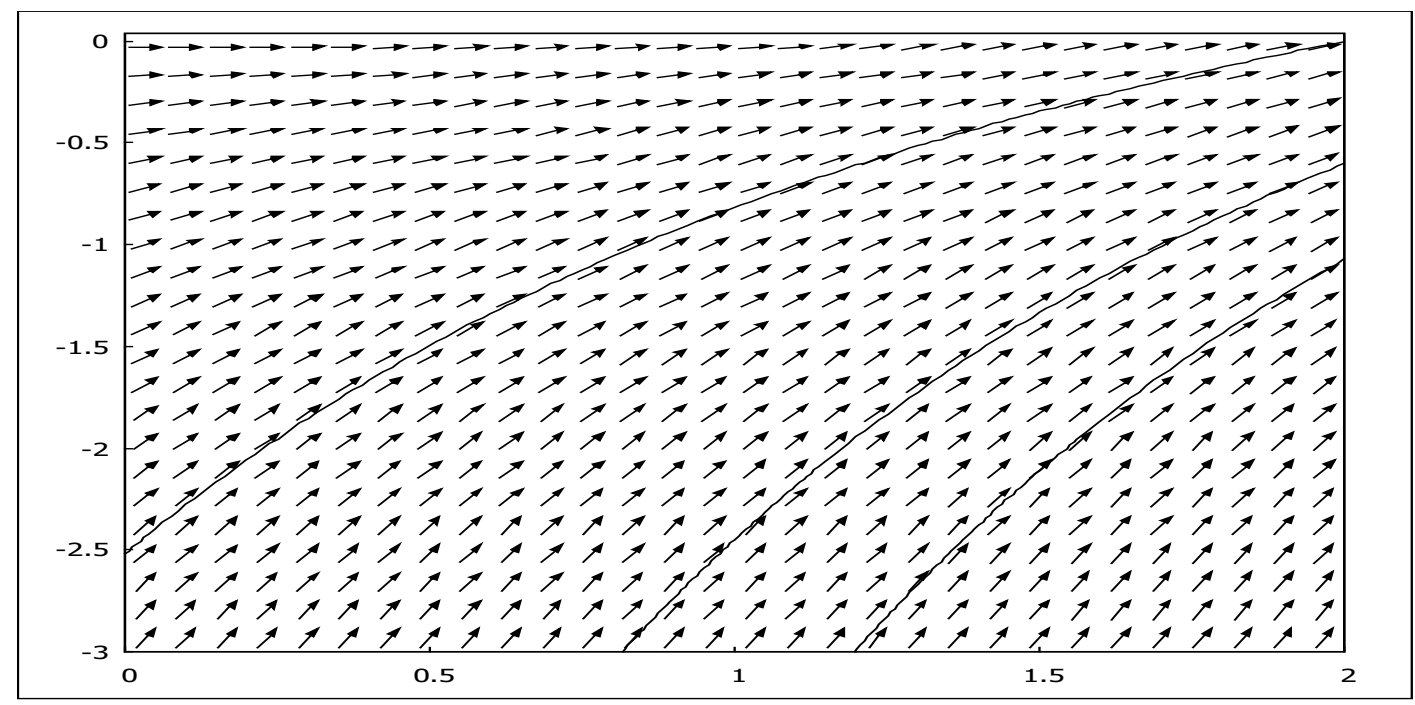

Fig. 1: The direction field of a differential equation and some solutions as paths representation tool

In order to estimate the error we consider the number of "groups" multiplied by a factor, namely $(6+9+4) \cdot 0.25 \mathrm{~s}=4.75 \mathrm{~s}$. This is coming from the transitions between the groups. The applied velocities for three and four persons were determined empirically, but not in this concrete simulation. All in all we have a second forecast: $t=39.7+4.75=44.45 \mathrm{~s}$ and recall the first one $\mathrm{t}=37.3 \mathrm{~s}$. An empirical plausibility control value $\mathrm{t}=37 \mathrm{~s}$ had been obtained and based on this time we have the flow rate 1.51 persons per second. The images in Fig 2 may offer an impression of the empirical data collection. Modelling the pedestrian flow applying any CA-model or macroscopic model would have been in fact more comfortable, but we do it on this way, bearing in mind future developments. We have actually simulated the physical part and calculated an evacuation time. Now, we dedicate our efforts to the psychological one. We proceed to implement one diagnostic test, including one hodological space. 


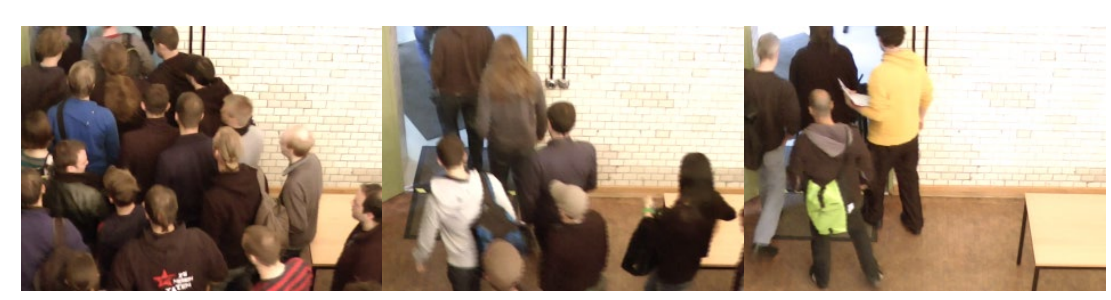

Fig. 2: From the left: the pedestrian flow beginning, in between and to the end

In this case we apply some knowledge of the group obtained during the event. Knowledge obtained before shall not be taken into account. For the complete simulation we thought of $90 \mathrm{~s}$ but we focus only on the bottleneck situation at the end and think of $45 \mathrm{~s}$. based on the mathematical simulation of the physical aspects. Because of the need for control concerning the shape of the flow applied in this model and the presence of a person on a wheelchair we set $\mathrm{T}=45$ and no subinterval division. A rather relaxed attitude due to more experience may induce a definition of subintervals, say breaks, while updating the values of the relevant variables:

Step 1 Time interval: $[0,45]$. (seconds)

Step 2 Relevant variables: A, attention paid to announcements (0 poor, 1 average, 2 highly concentrated), $\mathrm{C}$ social connectivity leading to clusters ( 0 none, 1 to one person, 2 to more than one person), Coh cohesion tendency in the group ( 0 no, 1 yes), D diffusion tendency in the group ( 0 no, 1 yes), M motor-perceptual region accessible ( 0 non-directly 1 directly), PA potential angriness ( 0 no, 1 yes)

Step 3 For the continuous interval: The answer to the question: What is possible? (Based on the actual situation) : No unexpected changes assumed

Step 4 For the continuous interval: proof the values of the relevant variables:

A: $18 \times 0,22 \times 1,16 \times 2$; C: $18 \times 0,32 \times 1,6 \times 2$; Coh: 1 x 0; D: 1 x 0; M: 1 x 0, 55 × 1; PA: 56 x 0;

Step 5 Sketch the hodological space: We have no breaks, we start at $\mathrm{t}=0$. Although Lewin describes the method to be used as approximation from the general topological space to the specific hodological one, we will construct the hodological space on another way, for the sake of a simpler, rather accessible presentation. At first we define a representation of one person and then we embed it into the model of the life space (hodological space) of this person:
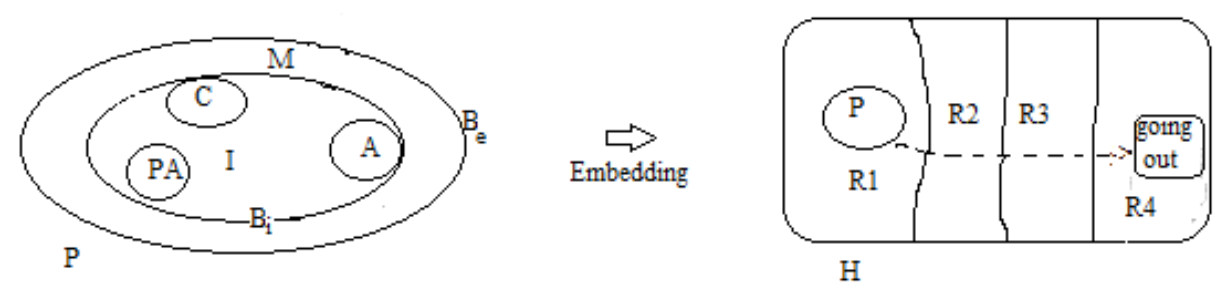

Fig. 3: The representation $\mathrm{P}$ of one person and its respective hodological space $\mathrm{H}$

In order to represent one person we introduce a set $\mathrm{P}$ including two subsets: the subset $\mathrm{I}$, representing the inner-personal region and the subset $\mathrm{M}$ representing the motor-perceptual activities. $\mathrm{B}_{\mathrm{e}}$ is the boundary between $\mathrm{P}$ and the environment. $\mathrm{B}_{\mathrm{i}}$ is the boundary between the motor-perceptual region and the inner-personal one.

As $\mathrm{M}$ includes information concerning the bodily interaction of the person and the environment, it is very important for the event management as it concerns the free accessibility of the event. In our example the 
subset $\mathrm{M}$ of one person representation includes the information wheelchair and assistance required. The value of the variable $M$ set to $M=0$, meaning non-directly accessible. For all other set $M=1$. We are aware of the use of $\mathrm{M}$ as subset name and as variable but we do it in order to simplify the notation. The subset I shall be poorly differentiated. Only the variables C (social connectivity), A (attention paid) and PA (potential angriness) are taken into account. As we see, Lewin's application of topology means on the one hand applying point set topology and on the other curves and no Venn-Diagrams for the graphical representation of psychological concepts. In the original papers a boundary is represented by a Jordan curve and the enclosed region is a set or subset. Then we embed $\mathrm{P}$ in a superset $\mathrm{H}$ and define subsets (regions) building a partition of $\mathrm{H}$. The partition might be coarse or fine, depending on what do we expect: if we do not expect social tensions or another kind of difficulties (an unexpected physical barrier or the behaviour of some person could produce potential angriness) then we set a coarse partition. We define a coarse partition because we do not expect any kind of tensions. Only because of the bottleneck situation we consider more than one subset, say region, to go through. One oriented path from $\mathrm{P}$ to the goal has been sketched. The choice of the expression "going out" instead of "exit" for the goal obeys the principle of underlining the fact we are dealing with the psychological and not with the physical world. As the distance in the hodological space is given by the number of regions to be crossed, it is obvious that fine partitions lead to longer distances. We have 56 such sets representing persons and we store the estimated variables values for each person at a given time. Now we define the group made out from all persons. Formally we could simply build the union set $\mathrm{G}$ including the 56 subsets, but we may not forget, following again Lewin, the group is not simply the union set but a different entity. Therefore, we define a set $\mathrm{G}$ inheriting all information included in the 56 sets representing persons, include some other subsets and embed it in the respective hodological space:
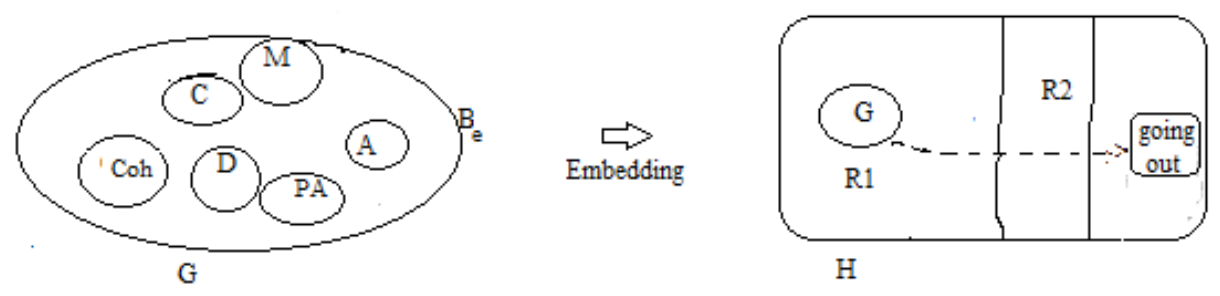

Fig. 4: The representation $\mathrm{G}$ of the group and its respective hodological space $\mathrm{H}$

The subsets C, M, A, PA include all information from the individual subsets. The boundary B separates again the set $\mathrm{G}$, the psychological world of the group, from the environment. The common intension of all persons to take part in the event builds up the group and defines also the boundary. We include the new variables Coh and $\mathrm{D}$ giving account of cohesion and diffusion tendencies in the group as a whole. $\mathrm{C}$ or Coh could show clustering tendencies. Changes in C, D or Coh could deal to a change of the parameter density, independently of the model. For the hodological space we define a partition which is coarser than the one defined in the individual example given above because we do not expect any complicated situation and because of the reduced number of choices for a behaviour change in case of the group.

Step 6 Return to Step 3 We recall: attempt to find an answer to the question: What is possible? (Based on the actual situation) In our example this leads to a continuous update of the variables values and of the partition of the hodological space.

In practice, in order to sketch the hodological space we could have already a template based on a data basis and/or empirical knowledge. The values of the variables is rather difficult to obtain. Start values 
may be taken from empirical data collections and adjusted by inspection. The values assumed here are taken from empirical knowledge. It is important to remark: no mean values should be taken as basis for decisions. In some cases (M) only once the value 1 and 55 the value 0 leads to an entirely different situation. Empirical if possible, but any way highly specific diagnostic analysis should be performed in order to make decisions. In our example, the observed and updated values did not lead to new calculations in the physical model.

\section{Introducing Entropy}

There are concepts of entropy applied to human sciences as Bailey's Social Entropy Theory, or von Cube's Social Group Entropy, which we do not include as reference in this paper because they play no role in our social event entropy concept. They find a mention at this place only for the sake of the interested reader. At this point seems to be adequate to say, that in this section we distinguish between a social event and an event as subset of a probability space. We shall construct the social event entropy concept directly and based exclusively on Shannon's theory [12].

Shannon's mathematical theory of communication was developed in the context of the fundamental problem of communication and from the point of view of engineering: reproducing a message independently of semantics. In contrast to the irrelevance of semantics we have: "the significant aspect is that the actual message is one selected from a set of possible messages" [12]. We proceed to present the definition of a communication system and then we apply a part of it to our aim, namely, the construction of a suited mathematical model of a social event which allows the definition of entropy.

A communication system is a system consisting of five parts: information source, transmitter, channel, receiver and destination. The information source produces a message and is the only part of importance to us. Following an interdisciplinary point of view, we introduce informally the mathematical model of the information source, as Shannon does. The mathematical description of an information source is a stochastic process, a discrete Markov process. Imagine a discrete information source as a message generator which generates a message symbol by symbol. The symbols are chosen, step by step, constrained by some probabilities which depend, on the one hand on the previous choice and on the other on the particular symbol. Quoting Shannon we state: "A physical system or a mathematical model of a system which produces such a sequence of symbols governed by a set of probabilities, is known as a stochastic process". In order to present a formal definition we should introduce a set $X_{t}$ of discrete random variables. The idea is to consider a social event as an information source producing messages. The messages we are interested on are, on the one hand, made out of quantities from the physical world and other hand made out of quantities from the psychological world. From the physical world we think of a five-dimensional vector with the components $t$, time, $x(t), y(t)$, position coordinates, $v(t)$ velocity magnitude and $\varphi(t)$ velocity angle. Formally, we should consider the product of some spaces, but in this paper we will not develop the theory in full strength. We just focus, in order to illustrate the idea, on the component velocity magnitude. So we consider the velocity magnitude as a value of a random variable attached to a person or a group at a given time. Then we find out the corresponding probability and calculate the entropy for the given time. For more details concerning the following entropy definition see [12]. The only slight difference between this definition and the classical one is the time aspect. We have for each visitor or group of visitors a probability at a given time. K might be used as norming factor. Any base may be applied but we prefer sometimes the natural base because the velocity, as function of the density, is an exponential function for this base. We write:

$$
H_{t}=-K \sum_{i=1}^{n} p_{i t} \log _{b} p_{i t}
$$

It is easy to calculate entropies. Therefore, we close this paper with one example and an application. If we have $\mathrm{N}=18$ visitors and the velocity is uniformly distributed, then we obtain in computer simulations almost always 18 different values. Therefore, the entropy with $\mathrm{K}=1$ will be $18(1 / 18) \cdot \log _{2}(18)=2.89$, the maximal value. If we set 18 times the same velocity we obtain $\mathrm{H}=0$. If we set 7 groups having 7 different 
velocities and the group sizes are 3, 6, 5 and 4 members plus 4 one person groups we obtain $H=1.66$. Vice versa, for a given entropy, we could imagine a group structure, as well in the psychological world.

\section{Conclusion}

The introduction of a systematic diagnostic analysis attempts to offer some answers to questions due to the interdependence of the physical and the psychological world concerning event management. Many of the problems are solved in practice by experienced event managers. Our concern is to improve mathematical models of human behaviour. As the example showed we obtain always a unified overview. There are methods for the analysis of the effect of uncertainty in an evacuation process [11]. The social event entropy may find an application field because of the level on which it works. The level is a kind of meta-level allowing uncertainty measurements on the physical and on the psychological world.

\section{Acknowledgements}

The author would like to acknowledge the contributions of Siegfried Paul, Arnim Bautz and students at the Beuth-Hochschule für Technik Berlin for making possible the empirical simulation.

\section{References}

[1] M. Chraibi, A. Schadschneider and A. Mackens, "Quantitative Description of Pedestrian Dynamics with a Force-Based Model", arXiv: 0910.2343v1 [physics.soc-ph], 2009.

[2] D. Helbing and P. Molnar , "Social Force Model for Pedestrian Dynamics," in Physical Review E, vol.51 (5), pp. 4282-4286, 1995.

[3] H. Klüpfel, T. Meyer-König and M. Schreckenberger, "Models for crowd movement and egress simulation”, in Proceedings of Traffic and Granular Flow '03, Berlin, 2004a, pp. 357-372.

[4] K. Lewin, Principles of Topological Psychology, New York: McGraw-Hill, 1936.

[5] K. Lewin, Field Theory in Social Sciences, New York: Harper \& Brothers, 1951.

[6] K. Lewin, "Defining the field at a given time (1941)", in Psychological Review 50(3), pp. 292-310, 1989

[7] J. Méndez Omaña, "Field Theory and Quantitative Models for Pedestrian Dynamics", in Transportation Research Procedia, vol. 2, pp. 442-445, 2014

[8] W.M. Predtetschenski and A.I. Milinski, Personenströme in Gebäuden, Berechnugsmethoden für die Projektierung, Köln-Braunsfeld: Rudolf Müller, 1971.

[9] A. Schadschneider and A. Seyfried, "Validation of CA Models for Pedestrian Dynamics with Fundamental Diagrams", in Cybernetics and Systems: An International Journal, vol. 40 5, pp. 367-389,

[10] I. von Sievers, A. Templeton, F. Künzner, G. Köster, J. Drury, A. Philippides, T. Neckel and H. Bungartz, "Modeling social identification and helping in evacuation simulation", in Safety Science, vol. 89, pp. 288-300, 2016.

[11] C. E. Shannon, "A Mathematical Theory of Communication", in The Bell System Technical Journal, vol. 27, pp. 379-423, 623-656, 1948.

[12] N. Wiener, Cybernetics: Or control and Communication in the Animal and the Machine, Cambridge MA: M.I.T. Press, 1948. 